\title{
MAGNETIC FIELD AND TURBULENCE IN SUNSPOTS
}

\author{
JEAN RAYROLE \\ Observatoire de Paris-Meudon, France
}

\begin{abstract}
We study the local variations of $\eta_{0}$ and $\xi$ (Milne-Eddington model) which are necessary in order to bring in agreement three independent determinations of the total field $H$, obtained from simultaneous observations of the two lines $\lambda 5250,2 \mathrm{Fe} \mathrm{I}$ and $\lambda 5225,5 \mathrm{Fe} \mathrm{I}$. The distribution of the broadening as a function of field direction is consistent with the motions induced by hydromagnetic waves.
\end{abstract}

\section{Introduction}

Observations of turbulent velocities in sunspots show that they are comparable with those in the undisturbed photosphere. The physical nature of these motions is an interesting problem since the magnetic field in sunspots may control the motions to a large extent. The low temperature of sunspots has been explained by Biermann (1941) as being caused by the inhibition of convection in a strong magnetic field. The possibility that travelling hydromagnetic waves are an important mode of energy transport has led to calculations on the generation of these waves in thermally unstable layers. De Jager (1964) shows that near a critical level ' $h c$ ' at a depth of five to ten thousand $\mathrm{km}$ (depending on the magnetic field) the convective motions are transformed into hydromagnetic and sound waves. The greater part of these hydromagnetic waves is reflected downward and only sound waves can reach the surface. Danielson (1965) shows that hydromagnetic waves can be emitted from regions above ' $h c$ '. Musman (1967) and Savage (1969) have computed a model in which Alfvén waves generated in convectively unstable layers are permitted to propagate upward. The broadening of spectral lines by Alfvén waves has been computed by Maltby (1968).

In this work we study in a large penumbra the local variations of saturation and Doppler broadening ( $\eta_{0}$ and $\xi$ in Milne-Eddington model) and their correlations with the field direction.

\section{The Material}

For a large sunspot (October 23, 1969 - N10-E31) we have obtained simultaneous spectrograms with right and left circular polarisation for the two lines of equal excitation potential:

$$
\begin{array}{ll}
\lambda 5250.22 \mathrm{Fe}_{\mathrm{I}} & \text { Zeeman pattern }(0,00) 3,00 \\
\lambda 5225.53 \mathrm{Fe} \mathrm{I} & \text { Zeeman pattern }(1,50) 1,503,00
\end{array}
$$

The different spectra (about 100) cover the sunspot with $1^{\prime \prime}$ resolution, and their effective resolution is about $2-3^{\prime \prime}$. However most of the data points discussed below belong to the penumbra of the large spot or to minor satellite umbrae, since in the largest umbra many points were underexposed. 


\section{Data Reduction}

The method used is a refinement of the one described by Rayrole (1967).

Let us introduce the following parameters.
$O X \quad$ direction of spectrograph slit;
$O Y \quad$ scanning direction $(\perp$ to $O X)$;
$\left.\begin{array}{l}F_{1} \\ F_{2}\end{array}\right\} \quad$ slits of the Lambdameter;
$\delta \lambda \quad$ width of the slits $F_{1}$ and $F_{2}$;
$\Delta \lambda \quad$ distance between the slits $F_{1}$ and $F_{2}$;
$\gamma \quad$ contrast factor of the photographic plate;
$R I_{R}(\lambda)$ depression of the line for right circular polarisation;
$R I_{L}(\lambda) \quad$ depression of the line for left circular polarisation.

For each $(X, Y)$ position on the spot and each observed line the lambdameter gives us the $\lambda_{R}(X, Y)$ and $\lambda_{L}(X, Y)$ wavelength for which respectively:

and

$$
\int_{\boldsymbol{F}_{1}}\left(1-R I_{R}(\lambda)\right)^{-\gamma} \mathrm{d} \lambda=\int_{\boldsymbol{F}_{2}}\left(1-R I_{R}(\lambda)\right)^{-\gamma} \mathrm{d} \lambda
$$

$$
\int_{F_{1}}\left(1-R I_{L}(\lambda)\right)^{-\gamma} \mathrm{d} \lambda=\int_{F_{2}}\left(1-R I_{L}(\lambda)\right)^{-\gamma} \mathrm{d} \lambda .
$$

Thus we introduce in a computer the four following parameters:

$E 251(X, Y)=\lambda_{R}(X, Y)-\lambda_{L}(X, Y)$ for $\lambda 5225, \delta \lambda=40 \mathrm{~m} \AA, \Delta \lambda=40 \mathrm{~m} \AA$ $E 253(X, Y)=\lambda_{R}(X, Y)-\lambda_{L}(X, Y)$ for $\lambda 5225, \delta \lambda=120 \mathrm{~m} \AA, \Delta \lambda=120 \mathrm{~m} \AA$ $E 501(X, Y)=\lambda_{R}(X, Y)-\lambda_{L}(X, Y)$ for $\lambda 5250, \delta \lambda=40 \mathrm{~m} \AA, \Delta \lambda=40 \mathrm{~m} \AA$ $E 503(X, Y)=\lambda_{R}(X, Y)-\lambda_{L}(X, Y)$ for $\lambda 5250, \delta \lambda=120 \mathrm{~m} \AA, \Delta \lambda=120 \mathrm{~m} \AA$

and we compare it with calibration curves $F\left(\eta_{0}, \xi, \beta_{0}, H, \psi\right)$ derived from theoretical profiles computed with Unno theory (Unno, 1956) and a Milne-Eddington model with:

$$
\begin{aligned}
& \eta=\eta_{0} e^{-v^{2}}+\eta_{0} a H_{1}(v)+\eta_{0} a^{2} H_{2}(v) \\
& v=\frac{\lambda-\lambda_{0}}{\xi} \quad a=\frac{\Gamma}{4 \pi \Delta v_{0}} \quad \Delta v_{0}=\frac{v_{0}}{c} \sqrt{\frac{2 K T}{M_{0} A}+v_{t}^{2}} \quad \Gamma=4 \cdot 10^{9} \mathrm{~s}^{-1}
\end{aligned}
$$

and $B(\tau)=B_{0}\left(1+\beta_{0} \tau\right)$ for the source function variation with optical depth.

In this way $E 251(X, Y)$ and $E 253(X, Y)$ give us the angle with the line of sight $\psi\left(X, Y, \eta_{0}, \xi\right)$ and the total field $H 25\left(X, Y, \eta_{0}, \xi\right)$ while $E 501(X, Y), E 503(X, Y)$ associated with the $\psi$ determination give us two other independent values of the total field $H 501\left(X, Y, \eta_{0}, \xi\right)$ and $H 503\left(X, Y, \eta_{0}, \xi\right)$. The local variations $\eta_{0}(X, Y)$ and $\xi(X, Y)$ are selected in order to bring in agreement the three determinations of the total field $H$. We take as a test of interval coherence, and therefore as indication for 
the choice of an optimal model $\left(\eta_{0}, \xi\right)$ the mean square root value $\operatorname{ECQM}\left(X, Y, \eta_{0}, \xi\right)$ of these three $H$ determinations.

$$
\begin{aligned}
& \operatorname{ECQM}\left(X, Y, \eta_{0}, \xi\right) \\
& =\sqrt{\frac{(H 25-H M)^{2}+(H 501-H M)^{2}+(H 503-H M)^{2}}{9}}
\end{aligned}
$$

where

$$
\begin{aligned}
& H M\left(X, Y, \eta_{0}, \xi\right) \\
& \quad=\frac{H 25\left(X, Y, \eta_{0}, \xi\right)+H 501\left(X, Y, \eta_{0}, \xi\right)+H 503\left(X, Y, \eta_{0}, \xi\right)}{3} .
\end{aligned}
$$

In each $(X, Y)$ position the $\eta_{0}$ and $\xi$ values are chosen in order to bring the ECQM function to a minimum.

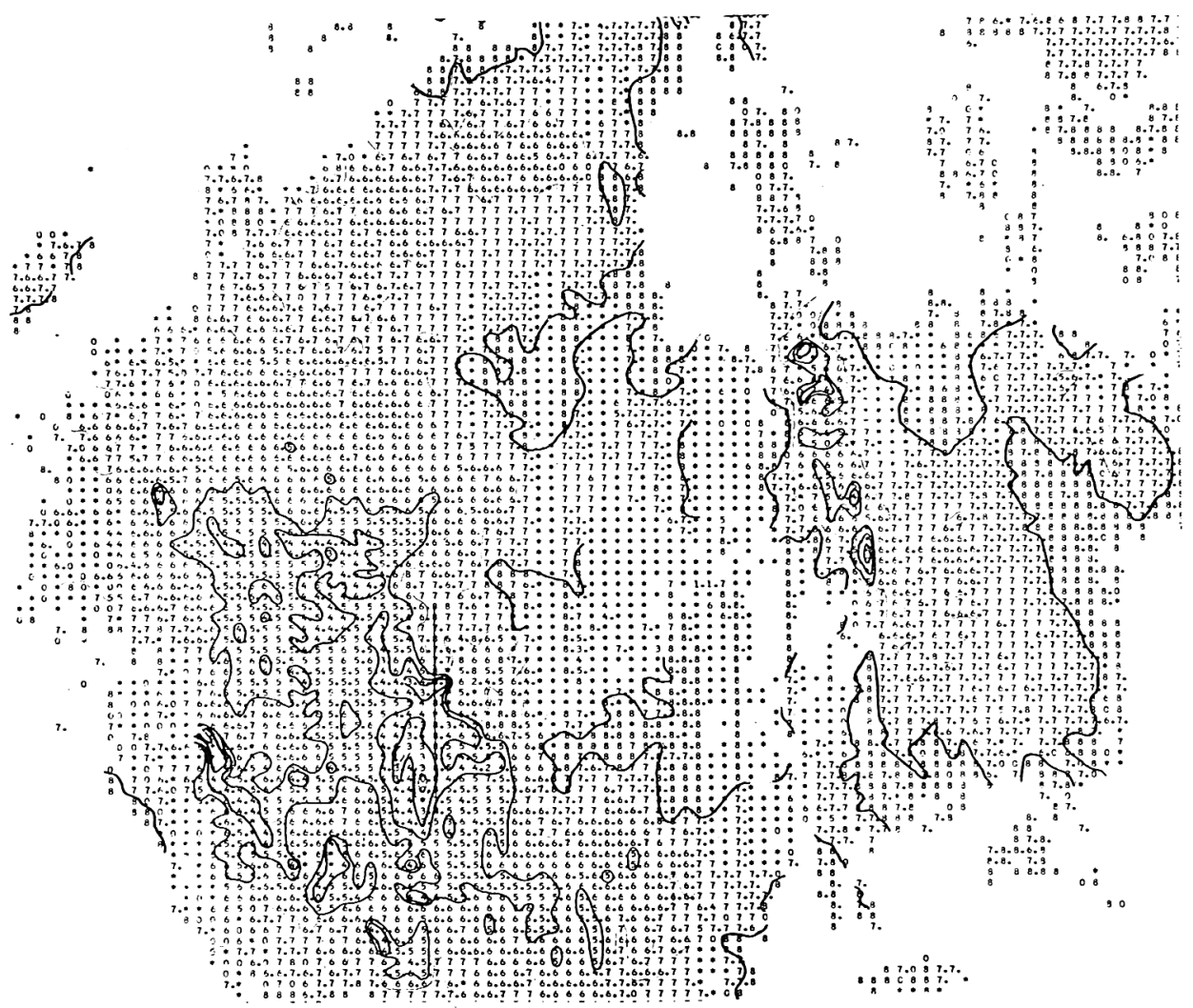

Fig. 1a. Map of the angle $\Psi$ between the field and the line of sight for Model 1. The distance between two points is $1^{\prime \prime}$. The $\Psi$ values are given by the following symbols:

Symbols

$0 \quad 0 . \quad 1$

1. 2 2. ...)

$\begin{array}{llllllll}\Psi \text { greater than (degrees) } & 0 & 5 & 10 & 15 & 20 & 25 & \ldots ;\end{array}$ and so on. 


\section{Observational Results}

In a first approach we have taken only three models characterized by

$$
\begin{array}{lll}
\text { Model 1 } & \eta_{0}=1 & \xi=40 \mathrm{~m} \AA \\
\text { Model 2 } & \eta_{0}=20 & \xi=40 \mathrm{~m} \AA \\
\text { Model 3 } & \eta_{0}=20 & \xi=20 \mathrm{~m} \AA
\end{array}
$$

Model 1 represents the undisturbed photospheric conditions while Models 2 and 3 are in good agreement with the great saturation given by an empirical model of the umbra (Henoux, 1969), and represent respectively the conditions where the turbulent velocities are the same as those of the undisturbed photosphere or only due to thermal motions.

Figure 1 shows the values of $\psi(X, Y)$ for the three models and therefore the great influence of $\eta_{0}$ and $\xi$ on the determination of the angle $\psi$ with line of sight. The differences between the $H$ determinations are shown in Figures 2, for a small region.

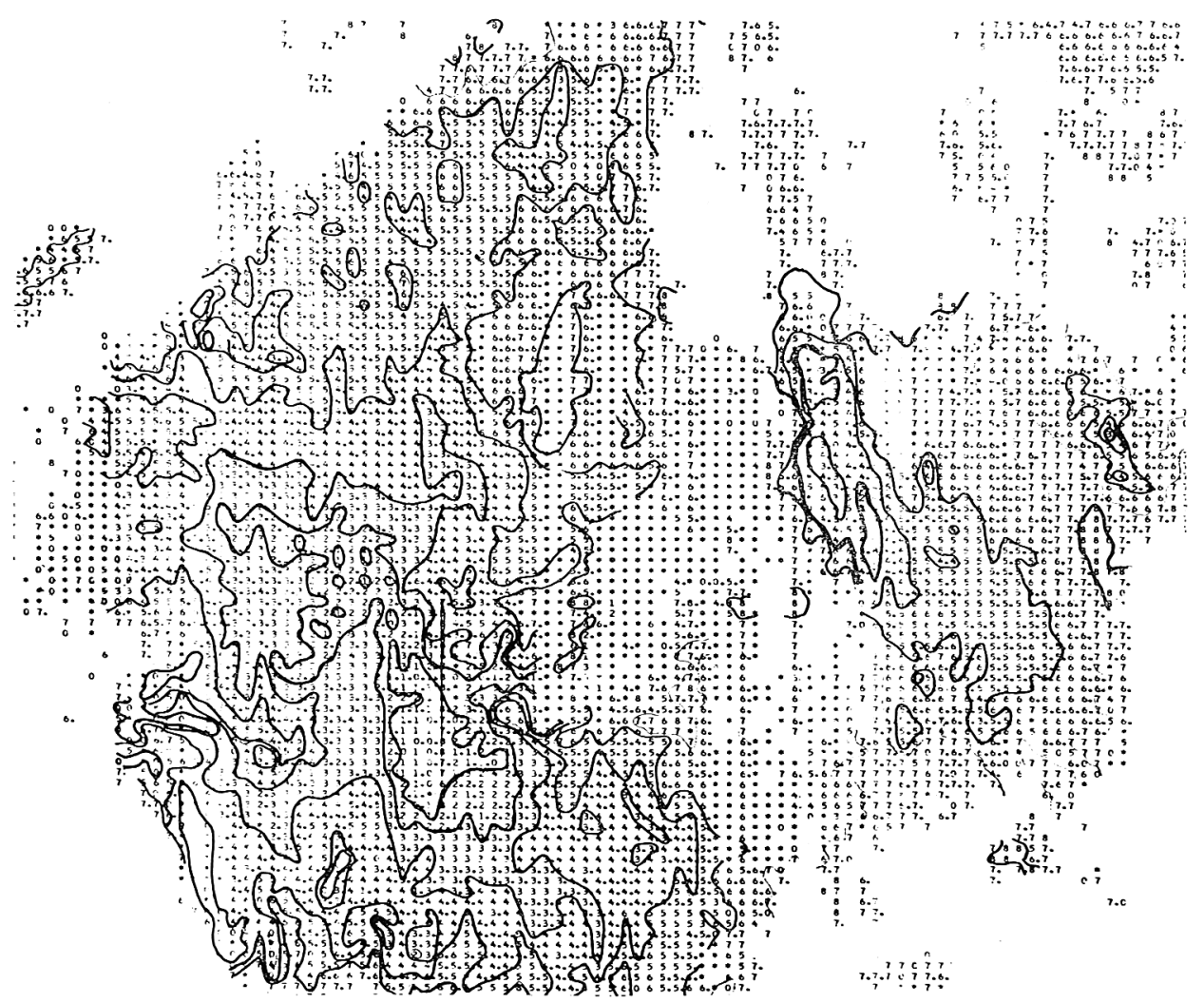

Fig. 1b. Map of the angle $\Psi$ between the field and the line of sight for Model 2. The symbols are the same as in Figure 1a. 


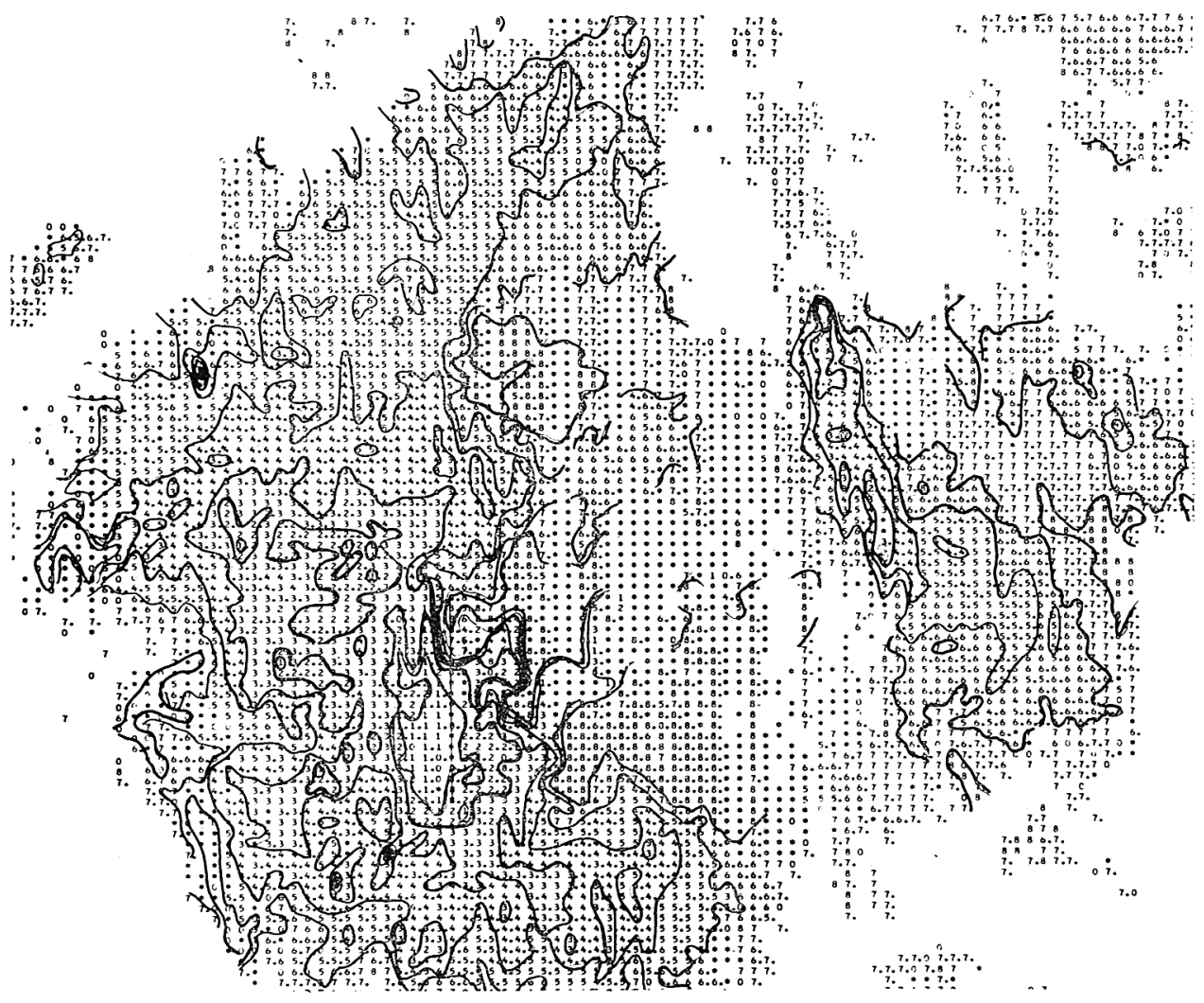

Fig. 1c. Map of the angle $\Psi$ between the field and the line of sight for Model 3. The symbols are the same as in Figure 1a.

We found that for $70 \%$ of the 4000 measured points the minimum of ECQM is less than the noise for only one of the three models. The distribution of the most suitable model is shown in Figure 3. For all the points where ECQM is less than the noise a statistical study gives

$$
\begin{array}{llll}
\text { Model 1 } & \eta_{0}=1 & \xi=40 \mathrm{~m} \AA & 21 \% \text { of measured points. } \\
\text { Model 2 } & \eta_{0}=20 & \xi=40 \mathrm{~m} \AA & 14 \% \text { of measured points. } \\
\text { Model 3 } & \eta_{0}=20 & \xi=20 \mathrm{~m} \AA & 64 \% \text { of measured points. }
\end{array}
$$

Therefore for the majority of measured points the turbulent motions are weak and perhaps negligible, since $\xi=20 \mathrm{~m} \AA$ describes the thermal motions for $\lambda 5250$ and $T=$ $3700 \mathrm{~K}$.

If the only contribution to the line broadening, except the thermal motions, is due to travelling hydromagnetic waves this effect must depend on the field direction. The percentage of measured points leading to the weak broadening (Model 3) and the large broadening (Model $1+2$ ) is shown in Figure 4 , as a function of $\psi$. 


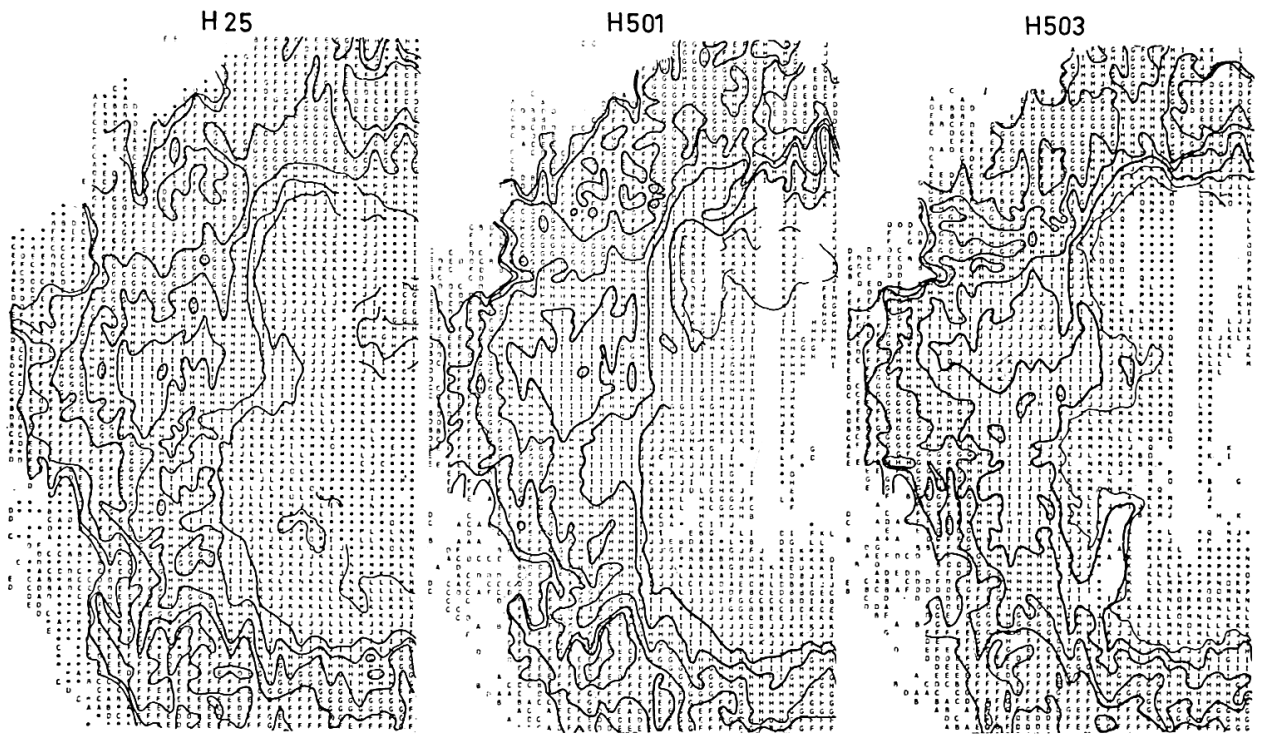

Fig. 2a. Map of the three $H$ values of the total field for Model 1. The distance between two points is $1^{\prime \prime}$. The $H$ values are given by the following symbols:

$\left.\begin{array}{llllll}\text { Symbols } & \text { A } & \text { B } & \text { C } & \text { D } & \text { E } \\ H \text { greater than (gauss) } & 200 & 400 & 600 & 800 & 1000\end{array}\right\}$ and so on.
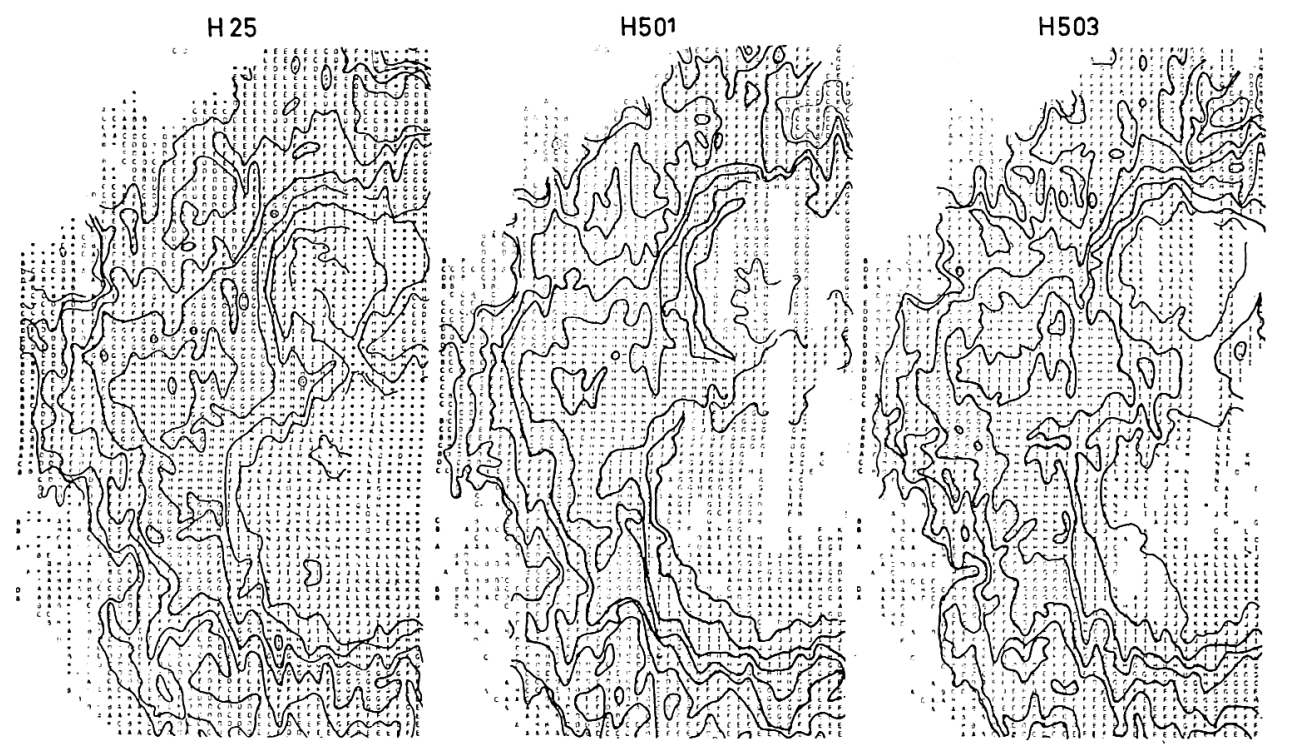

Fig. 2b. Map of the three $H$ values of the total field for Model 2. The symbols are the same as in Figure 2a. 

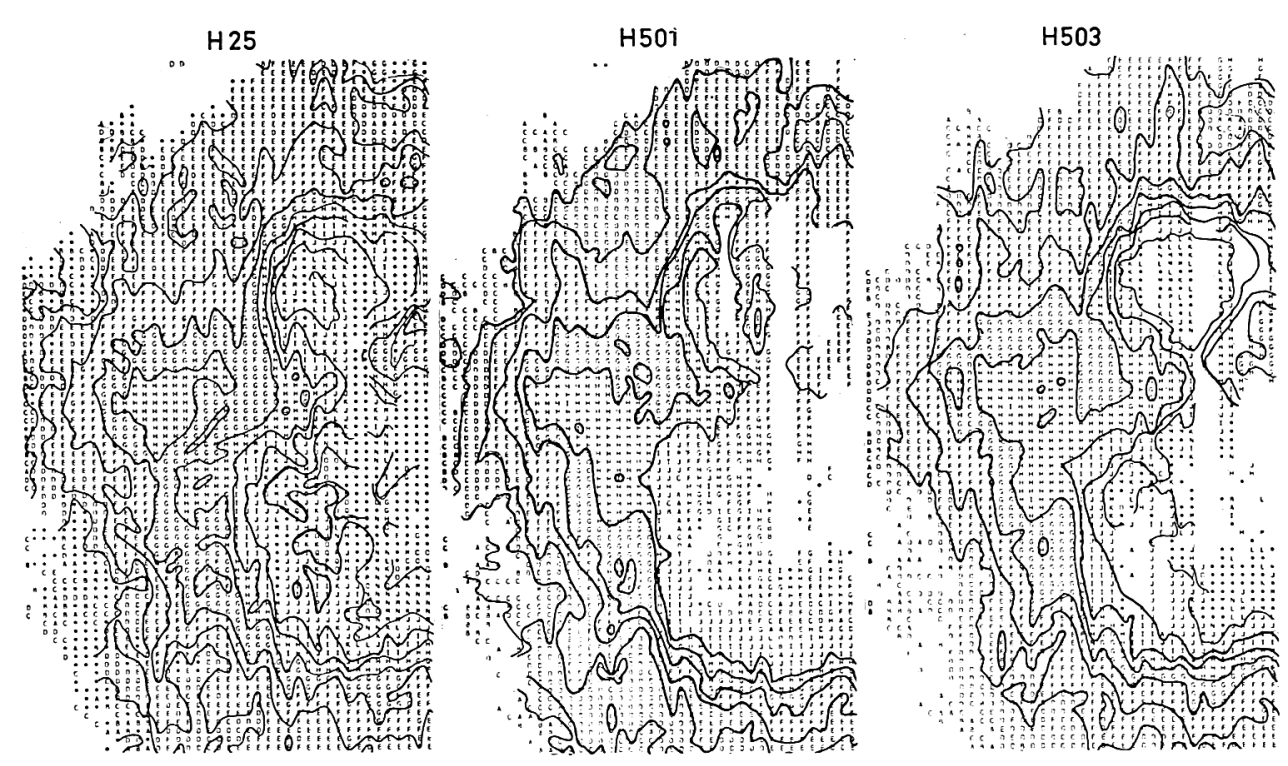

Fig. 2c. Map of the three $H$ values of the total field for Model 3. The symbols are the same as in Figure 2a.

Clearly large turbulent motions are associated with large $\psi$ values, while at low $\psi$ the profiles are generally consistent with no appreciable turbulent broadening. At very small $\psi\left(<15^{\circ}\right)$ however it becomes impossible to select one model rather than another because the calibration functions $F$ become insensitive to the model $\left(\eta_{0}, \xi\right)$.

The above correlation is consistent with the motions induced by Alfvén waves, or fast magnetosonic waves since $V_{a}=H /(4 \pi \varrho)^{1 / 2}$ is greater than the sound velocity in sunspots. The tendency to equipartition of the different models for great angles can be explained by the fact that:

(a) We have only taken three models to reduce the data.

(b) In an inhomogeneous atmosphere (Cowling, 1957) the vertical component of the motions induced by hydromagnetic waves is rapidly suppressed if $\delta \rho / \delta z$ is great enough. In this case the motions are horizontal and cannot produce a broadening when the field is in the vertical plane containing the line of sight.

\section{Conclusion}

The results obtained in this work bring us to make the following remarks.

(a) For the study of fine structures of magnetic field in sunspots it is not possible to discard the line broadening variations.

(b) This first approach points out possible observational evidence for the existence of hydromagnetic waves in sunspots. However a more accurate reduction with numerous models is needed to study the distribution of the broadening as a function of the 


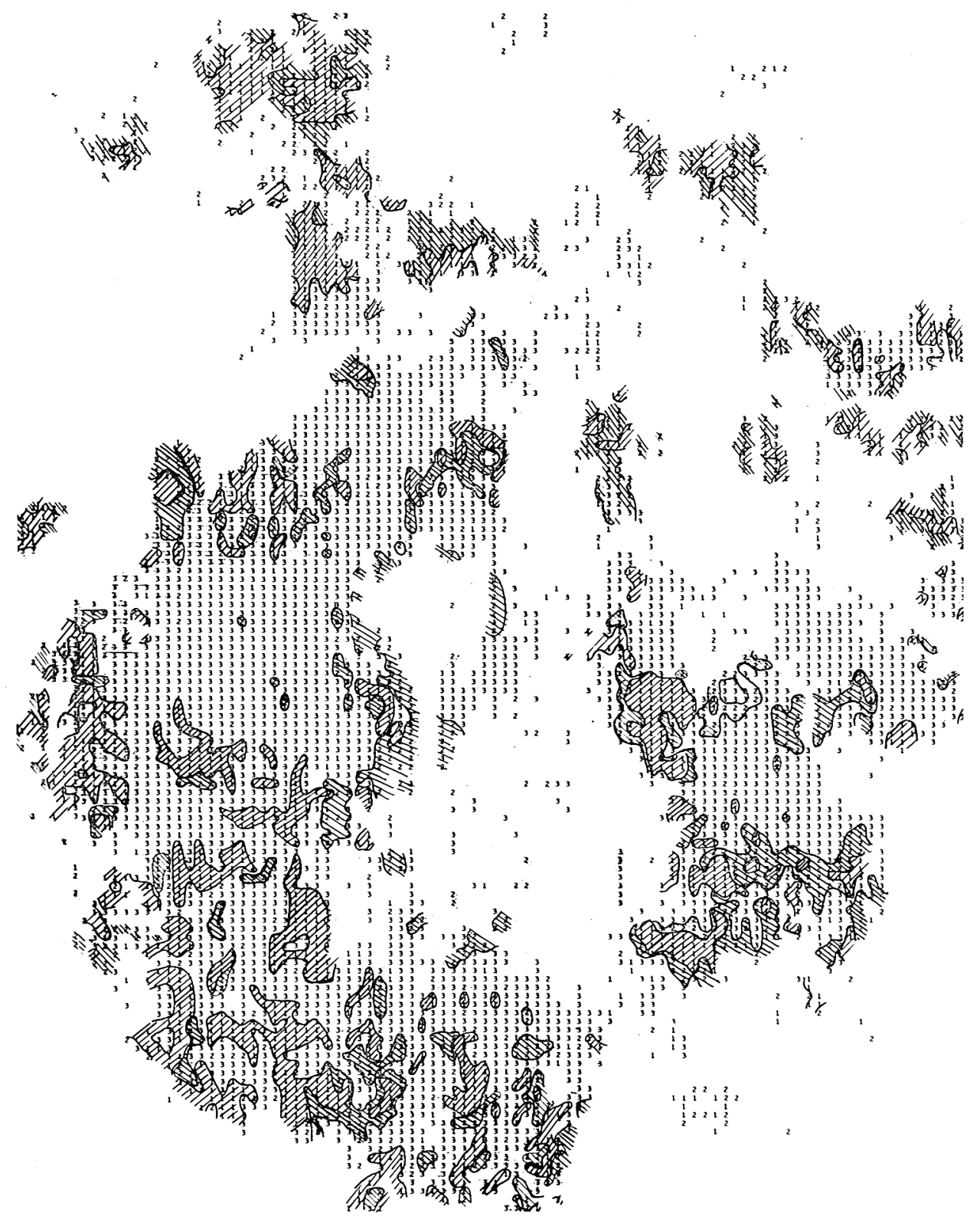

Fig. 3. Distribution of the three Models $1,2,3$ as a function of the $X, Y$ positions. The distance between two points is $1^{\prime \prime}$. 


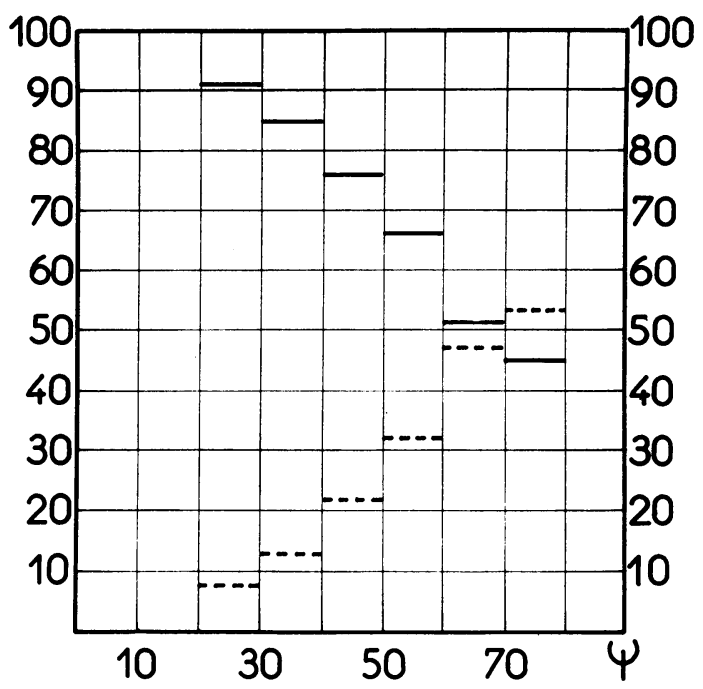

Fig. 4. Percentage of the models as a function of $\Psi$. —_ Model 3, weak broadening; -...- Model 1 +2 , large broadening.

field direction. It will be necessary to separate more definitely the relative influences of variations in saturation and broadening.

\section{Acknowledgements}

The author wishes to thank Mrs Savinelli for help with the lambdameter reduction.

\section{References}

Biermann, L.: 1941, Vierteljahresschr. Astron. Ges. 76, 194.

Cowling, T. G.: 1957, Magnetohydrodynamics, Interscience Publishers, New-York.

Danielson, R. E.: 1965, in R. Lüst (ed.), 'Stellar and Solar Magnetic Fields', IAU Symp. $22,314$.

De Jager, C.: 1964, Bull. Astron. Inst. Neth. 17, 253.

Henoux, J. C.: 1969, Astron. Astrophys. 2, 288.

Maltby, P.: 1968, Solar Phys. 5, 14.

Musman, S.: 1967, Astrophys. J. 149, 201.

Rayrole, J.: 1967, Ann. Astrophys. 30, 257.

Savage, B..D.: 1969, Astrophys. J. 156, 707. 